\title{
Primary biliary cirrhosis in a rheumatoid arthritis patient treated with rituximab, a case-based review
}

\author{
Joaquim Polido-Pereira • Ana Maria Rodrigues • \\ Helena Canhão • Fernando Saraiva • \\ José Alberto Pereira da Silva • João Eurico Fonseca
}

Received: 6 September 2011 /Revised: 26 September 2011 /Accepted: 12 October 2011

(C) Clinical Rheumatology 2011

\begin{abstract}
Primary biliary cirrhosis (PBC) is an autoimmune disease in which intrahepatic bile ducts are targeted by an immune-mediated injury. This disease tends to progress to fibrosis and cirrhosis with hepatic failure. The authors report a case of a 50-year-old rheumatoid arthritis (RA) patient, with erosions and seropositive for rheumatoid factor and anti-citrullinated peptide antibodies, with 18 years disease duration refractory to prednisolone and several disease-modifying antirheumatic drugs, either conventional or biological (adalimumab and etanercept). In April 2007, she started therapy with rituximab (RTX) with good European League Against Rheumatism response achieved 9 months later. In June 2008, she was admitted with intrahepatic cholestasis, steatorrhea, and spontaneous fractures of various ribs. After excluding cholelitiasis, as well as infectious and neoplastic diseases a liver biopsy was performed that was compatible with the diagnosis of PBC. The antinuclear antibodies (1/160) were positive as well as the antimitochondrial antibodies (1/640). Other antibodies were negative such as anti-SSA and anti-SSB. Afterwards, the patient started ursodesoxycholic acid $15 \mathrm{mg} \mathrm{kg}^{-1}$ day $^{-1}$ with progressive improvement of cholestatic markers. A labial salivary gland biopsy was performed and showed findings compatible with the concomitant diagnosis of
\end{abstract}

\footnotetext{
J. Polido-Pereira $\cdot$ A. M. Rodrigues $\cdot$ H. Canhão $\cdot$ J. E. Fonseca Rheumatology Research Unit, Instituto de Medicina Molecular, Faculdade de Medicina, Universidade de Lisboa, Lisbon, Portugal

J. Polido-Pereira $(\bowtie) \cdot$ A. M. Rodrigues $\cdot$ H. Canhão

F. Saraiva $\cdot$ J. A. P. da Silva $\cdot$ J. E. Fonseca

Rheumatology Department, Centro Hospitalar de Lisboa Norte, EPE, Hospital de Santa Maria,

Lisbon, Portugal

e-mail: polidopereira@gmail.com
}

Sjögren's syndrome. Based on this clinical report, a detailed review of the clinical aspects of $\mathrm{PBC}$ is presented as well as its association with other immune-mediated inflammatory diseases, particularly, with RA.

Keywords Biologic therapy · Primary biliary cirrhosis . Rheumatoid arthritis $\cdot$ Rituximab

\section{Introduction}

Primary biliary cirrhosis (PBC) is a chronic autoimmune liver disease characterized by the slow progressive destruction of small bile ducts leading to cholestasis. This chronic non-suppurative cholangitis is immune mediated by CD4+ and $\mathrm{CD} 8+\mathrm{T}$ cells, anti-mitochondrial antibodies, innate immunity, and $\operatorname{IgA}$ transcytosis.

$\mathrm{PBC}$ is more frequent in women and it is usually diagnosed by the fourth and fifth decades of life [1-3]. Common disease manifestations are asthenia, chronic pruritis, digestive malabsorption, xanthelasma, and jaundice. However, some studies suggest that in more than half of the patients, the diagnosis is established at an asymptomatic stage [4]. Other disease manifestations include metabolic bone disease, such as osteomalacia and osteopenia, which are likely secondary to the malabsorption. Lab manifestations include moderate to high elevation of alkaline phosphatase (ALP) and gamma glutamyltransferase (GGT), as well as mild transaminases elevation. Bilirubin is usually normal during the early phase of the disease. The presence of anti-mitochondrial antibodies increase the likelihood of the diagnosis, but liver histology is critical to ascertain the diagnosis. Ludwig and Scheuer established four histological stages: portal damage, periportal damage, septal damage, and cirrhosis [5]. Early 
diagnosis of this disease is of outmost relevance since early treatment with ursodeoxycholic acid has proven to be effective in decreasing liver-related mortality as well as the need for liver transplantation [6]. Of note, PBC can be associated with many other autoimmune diseases, such as Sjögren's syndrome (SS), Raynaud syndrome, autoimmune thyroid disease, and systemic sclerosis. In fact, $70 \%$ of the PBC patients have sicca syndrome [7-11].

The association of PBC with rheumatoid arthritis (RA) is less clear, although several studies since the 1970 s point to a possible association; however, the true prevalence of $\mathrm{PBC}$ in RA is not well known, and this may impose several therapeutic and diagnostic challenges as we will demonstrate in this paper $[12,13]$.

\section{Case report}

This case report refers to a 50-year-old patient who was diagnosed with RA in 1990, when she was 32 years old. She presented with 2 years disease duration of symmetric additive polyarthritis involving proximal interphalangeal and metacarpophalangeal joints, wrists, shoulders, knees, ankles, tarsus, and metatarsophalangeal joints, with over $2 \mathrm{~h}$ of morning stiffness. She was rheumatoid factor positive $\left(80 \mathrm{IU} \mu \mathrm{l}^{-1}\right)$ and had discrete erosions identified in some metacarpophalangeal joints after 2 years of disease duration. She also referred xerostomia, xerophtalmia, and occasional aphtous ulcers. Antinuclear antibodies (including SSA and SSB) were negative, and there was no evidence of keratoconjuctivitis sicca.

She was subsequently treated with several consecutive disease-modifying antirheumatic drugs (DMARDs), starting with sulphasalazine up to $1.5 \mathrm{gday}^{-1}$ that had to be stopped due to nausea and vomiting. She was also treated with prednisolone $5-10 \mathrm{mg} \mathrm{day}^{-1}$. In 1992, she started gold salts, but had to be interrupted in March 1994 due to aphtous stomatitis. In the meantime, several steroid intraarticular injections were performed (right wrist, second and third metacarpophalangeal joints). In 1995, she started therapy with methotrexate (MTX) up to $7.5 \mathrm{mg}^{\text {week }}{ }^{-1}$, and hydroxychloroquine (HCQ) $400 \mathrm{mg} \mathrm{day}^{-1}$ was added subsequently. MTX was also suspended in 1997 due to mucositis. As an alternative to MTX, azathioprine (AZA) was started in a dose up to $100 \mathrm{mg}$ day $^{-1}$ in December 1997. Despite this therapy, she maintained high disease activity (DAS28 of 6.38). She received concomitant treatment with calcium and vitamin $\mathrm{D}$, and as the bone densitometry showed a T-score of -2.26 in the hip and -1.98 in the lumbar spine, she was started on alendronate $70 \mathrm{mg} \mathrm{week}^{-1}$ in 2003 .

In April 2003, due to sustained high disease activity, she was medicated with adalimumab (ADA) $40 \mathrm{mg}$ every other week, maintaining AZA, HCQ, and prednisone. As the patient had only a partial response, AZA was replaced by MTX 7,5 mg week ${ }^{-1}$, in June 2004 . However, DAS28 was persistently above 5.2 , and she was switched to etanercept in March 2006. Unfortunately, the same pattern of high disease activity persisted under etanercept and she was then proposed for treatment with RTX 1,000 mg 2 weeks apart, which was started in April 2007. B cell depletion was confirmed. A year later, she was with low disease activity.

In June 2008, she was admitted due to a new onset asthenia, adinamia, and weight loss, as well as slight fever (maximum axillary temperature of $38^{\circ} \mathrm{C}$ ), steatorrhea, and an episode of spontaneous posterolateral right thoracic pain. The physical examination was unremarkable, except for several skin excoriations, and tender palpation of four consecutive right ribs (from fifth to eight). She had elevated GGT (268 IU $1^{-1}$, seven times upper limit of normal) and ALP (403 IU $1^{-1}$, four times upper limit of normal). ALP bone isoenzyme was only borderline high $\left(34.8 \mu \mathrm{g} \mathrm{I}^{-1} ; N<22.5\right)$, despite the fractures. Serum calcium, phosphorus, parathyroid hormone, TSH, and immune electrophoresis were normal. Cultures were negative. By this time, B cells had already repopulated peripheral blood. The chest radiograph showed fractures of four consecutive ribs. Abdominal ultrasound was compatible with hepatic steatosis. The investigation regarding occult neoplasia was negative and the patient was dismissed. At that time, a bone densitometry was repeated and showed a T score of -2.4 in the hip and -2.2 in the lumbar spine. A few months later, the patient was readmitted due to multiple fractures (including a fragility wrist fracture), progressive weight loss (about $20 \mathrm{~kg}$ in 6 months), severe steatorrhea, and cholestasis. Reevaluation showed a pseudopolypoid lesion of the duodenum. The biopsy was compatible with ganglioneuroma. However, this finding was not considered to be related with cholestasis, since the endoscopic retrograde cholangiopancreatography was normal and the abdominal ultrasound showed no biliary dilation. The patient had intrahepatic cholestasis, with very high ALP and GGT, mildly elevated levels of TGP and TGO, and elevated IgM. Serum calcium and phosphorus levels were again normal. These clinical and biochemical features were suggestive of $\mathrm{PBC}$, and a liver biopsy was performed which showed findings compatible with this diagnosis, Ludwig's stage 3 . Besides, at this time point, antimitochondrial antibodies were positive in high titer $(1 / 640)$, which further supported this hypothesis. She had also positive antinuclear antibodies (1/160), with negative antidouble stranded DNA (anti-dsDNA), anti-SSA, and antiSSB. Ursodiol therapy was started with progressive improvement of the cholestasis markers. A salivary labial 
biopsy was performed that showed focal lymphocytic sialadenitis, suggesting SS. A hand ultrasound was performed that confirmed the existence of erosions in several metacarpophalangeal joints and wrists and no synovitis; at that time, the patient was also anticitrullinated peptide antibodies (ACPA)-positive (178 EU). A couple of months later, the patient was submitted to partial duodenopancreatectomy, and the diagnosis of ganglioneuroma was confirmed.

Reviewing the patient chart, there were previous episodes of elevation of cholestatic markers (ALP and GGT) which were always self-limited, asymptomatic. and interpreted as drug-induced events.

In March 2010, due to disease reactivation, the patient was retreated with RTX, but this time without any clinical significant response. A further switch to abatacept was tried, but currently, RA remains active. CBP manifestations are quiescent.

\section{Discussion}

This case report illustrates the association of multiple immune-mediated diseases and the therapeutic challenges which this scenario arises. This patient had a classic, established RA with almost 20 years of disease duration and was exposed to multiple DMARDs, ending with RTX after failing two TNF inhibitors. In fact, there was a clinically significant response (good European League Against Rheumatism response), but when the patient was going to be retreated she was admitted due to severe cholestasis and multiple rib fractures. Chronic cholestasis seemed to explain bone fragility, but the atypical rib fractures forced the work out for an occult neoplasia. Bone metabolic disturbances induced by long-standing RA, chronic corticosteroid treatment, and prolonged exposure to biphosphonates might have contributed for the atypical pattern of bone fragility, initially dominated by multiple rib fractures. In the absence of biliary tree dilation, the investigation had to rely on causes of intrahepatic cholestasis. The clinical presentation and the fact that there were very high levels of ALP and GGT, mildly elevated AST and ALT, and high IgM suggested the diagnosis of PBC, which was confirmed after compatible serology and liver biopsy. After reviewing the patient's chart, there were previous episodes of cholestasis which seemed to be drug-induced; they were always mild to moderate and self-limited. After 1 year of RTX treatment, severe intrahepatic cholestasis surfaced, and interestingly, this event was coincident with B cell repopulation.

Hepatic involvement of most rheumatic diseases is common and usually related with nonspecific findings (drug-related most of the times). However, more serious hepatic involvement, including vasculitis, nodular regenerative hyperplasia, and primary biliary cirrhosis, have been observed in specific rheumatic diseases, such as RA $[14,15]$.

Furthermore, ALP elevations in RA are common and rarely related with $\mathrm{PBC}$ [16]. In a recent review of 607 patients with diffuse connective tissue diseases, liver dysfunction was observed in $38.2 \%$ and PBC was present in $15.9 \%$. This review showed that in scleroderma and SS, PBC was the cause of liver dysfunction in above $70 \%$ of the patients, which did not occur in RA where cases of PBC were rare ( 3 out of 220 patients) [17].

RA is present in about $1 \%$ of the population and $\mathrm{PBC}$ is even rarer, with an estimated prevalence of 20/100,000 for woman and 2/100,000 for men and the association of both diseases by chance is very unusual [10]. However, there are some reports describing its association in individuals or families $[18,19]$. Few studies have suggested an increased prevalence $(1.8 \%$ and $5.6 \%)$ of RA in PBC patients as compared to the prevalence in the general population $[4$, 20, 21]. In one series of 42 patients with PBC, four presented symptomatic arthritis with rheumatoid factor positivity, and nine had erosions without symptomatic arthritis [22]. In another series, 4 out of 83 patients with PBC presented inflammatory arthritis [23]. In a Japanese review, 3 of the 54 patients with $\mathrm{PBC}$ had polyarthritis, without specifying whether or not RA criteria were fulfilled [24]. In a 1980s review of 26 PBC patients, 7 were classified as RA, which seems an overestimation [25]. On the contrary, a recent review of 278 patients with autoimmune hepatitis (some of them overlapping with PBC), 111 had concurrent autoimmune diseases, of which only 5 had RA, according to the ACR 1987 ARA diagnostic criteria [26].

Currently, there is no accurate estimate of PBC prevalence in RA patients but several studies estimated that $10-18 \%$ of these patients have antimitochondrial antibodies, which have indeed a high association with PBC. Importantly, most of these patients had associated syndromes, as was the case of our patient. On the other hand, approximately half of the patients with PBC become rheumatoid factor positive during the course of the disease [27-29]. In fact, in an older series, $70 \%$ of the PBC patients were rheumatoid factor positive [8]. Serology overlap also happens with ACPA. In fact, according to Koga et al. ACPA are positive in $2.7 \%$ of the $\mathrm{CPB}$ patients and in $10.5 \%$ of the autoimmune hepatitis patients, almost always in association with a clinical presentation compatible with concomitant RA [30]. In another work, Santiago et al. showed that ACPA (third generation) were present in $3.7 \%$ of the $\mathrm{PBC}$ patients [31]. 
Despite the low prevalence of PBC in RA patients, auto antibodies overlap and shared genetic risk factors, namely, related with CTLA4 and ICOS SNPs, suggesting the existence of at least some common physiopathological mechanisms [32]. In fact, RTX has been proposed as a treatment option for $\mathrm{PBC}$ and curiously overt clinical and laboratorial manifestations of $\mathrm{PBC}$ occurred in our patient after B cell repopulation. Another interesting and unexplored field is the disturbance of costimulation that occurs in PBC that might benefit from costimulation modulating therapies such as abatacept [33, 34]. However, the use of abatacept in our patient (introduced for RA treatment) did not influence PBC clinical manifestations.

\section{Key points}

(1) $\mathrm{PBC}$ is a rare disease that can appear in association with several other immune-mediated diseases

(2) PBC's association with RA is unclear despite some reports in the literature suggesting a possible link between both diseases

(3) In patients with rheumatic diseases, persistent elevation of liver enzymes, mostly with cholestatic pattern, shall raise the suspicion of a possible PBC

Acknowledgments The authors thank Dr. Ana Afonso and Dr. Maria Adelaide Milheiro from the Pathology Department of Hospital Curry Cabral, Lisbon, for the clinical discussion and for sharing the pathology images.

Disclosures None.

\section{References}

1. Kaplan MM (2005) Primary biliary cirrhosis. N Engl J Med 353:1261

2. Nguyen DL, Juran BD, Lazaridis KN (2010) Primary biliary cirrhosis. Best Pract Res Clin Gastroenterol 24(5):647-654

3. Selmi, C, Gershwin E (2008) Primary biliary cirrhosis. Diagnostic criteria in autoimmune diseases, vol. 55. Humana Press, pp 291-294

4. Inoue K, Hirohara J, Nakano T et al (1995) Prediction of prognosis of primary biliary cirrhosis in Japan. Liver 15:70-77

5. Leuschner U (2003) Primary biliary cirrhosis - presentation and diagnosis. Clin Liver Dis 7:741-758

6. Poupon RE, Lindor KD, Cauch-Dudek K et al (1997) Combined analysis of randomized controlled trials of ursodeoxycholic acid in primary biliary cirrhosis. Gastrenterology 90:247-249

7. Gershwin ME, Selmi C, Worman HJ et al (2005) Risk factors and comorbidities in primary biliary cirrhosis: a controlled interview-based study of 1032 patients. Hepatology 42(5):11941202

8. Culp KS, Fleming CR, Duffy J et al (1982) Autoimmune associations in primary biliary cirrhosis. Mayo Clin Proc 57:365-370
9. Uddenfeldt P, Danielsson A, Forssell A et al (1991) Features of Sjögren's syndrome in patients with primary biliary cirrhosis. J Intern Med 230:443-448

10. Siegel JL, Luthra H, Donlinger J, Angulo P, Lindor K (2003) Association of primary biliary cirrhosis and rheumatoid arthritis. $\mathrm{J}$ Clin Rheumatol 9:340-343

11. Elta GH, Sepersky RA, Goldberg MJ et al (1983) Increased incidence of hypothyroidism in primary biliary cirrhosis. Dig Dis Sci 28:971-975

12. Child DL, Mathews JA, Thompson RP (1977) Arthritis and primary biliary cirrhosis. Br Med J 2(6086):557

13. Mills P, MacSween RN, Watkinson G (1977) Arthritis and primary biliary cirrhosis. Br Med J 2(6096):1224

14. Walker NJ, Zurier RB (2002) Liver abnormalities in rheumatic diseases. Clin Liver Dis 6(4):933-946

15. Abraham S, Begum S, Isenberg D (2004) Hepatic manifestations of autoimmune rheumatic diseases. Ann Rheum Dis 63:123129

16. Spooner RJ, Smith DH, Bedford D, Beck PR (1982) Serum gamma-glutamyltransferase and alkaline phosphatase in rheumatoid arthritis. J Clin Pathol 35(6):638-641

17. Takahashi A, Abe K, Yokokawa J, Iwadate H, Kobayashi H, Watanabe H, Irisawa A, Ohira H (2010) Clinical features of liver dysfunction in collagen diseases. Hepatol Res 40(11):10921097

18. Watt FE, James OF, Jones DE (2004) Patterns of autoimmunity in primary biliary cirrhosis patients and their families: a population based cohort study. QJM 97:397-406

19. Caramella C, Avouac J, Philippe S et al (2007) Association between rheumatoid arthritis and primary biliary cirrhosis. Joint Bone Spine 74:279-281

20. Marasi IB, Gagetta M, Rossi V et al (2001) Rheumatic disorders and primary biliary cirrhosis: an appraisal of 170 Italian patients. Ann Rheum Dis 60:1046-1049

21. Ely GM, Perruquet JL, Newman ED (1996) The arthritis of primary biliary cirrhosis: clinical features and associated immune processes. J Clin Rheumatol 2(4):191-196

22. Mills PR, Vallance R, Birnie G et al (1981) A prospective survey of radiological bone and joint changes in primary biliary cirrhosis. Clin Radiol 32(3):297-302

23. Clarke AK, Galbraith RM, Hamilton EB, Williams R (1978) Rheumatic disorders in primary biliary cirrhosis. Ann Rheum Dis 37(1):42-47

24. Takagi T, Kasukawa R (1994) Association of autoimmune disease in primary biliary cirrhosis. Nippon Rinsho 52(1):251255

25. Uddenfeldt P, Danielsson A (1986) Evaluation of rheumatic disorders in patients with primary biliary cirrhosis. Ann Clin Res 18(3):148-153

26. Teufel A, Weinmann A, Kahaly GJ et al (2010) Concurrent autoimmune diseases in patients with autoimmune hepatitis. J Clin Gastroenterol 44(3):208-213

27. Datta A, Deodhar SD, Datta U et al (1990) Non-organ specific and organ specific antibodies in rheumatoid arthritis. Indian J Med Res 92:228-232

28. Zurgil N, Bakimer R, Moutsopoulos HM et al (1992) Antimitochondrial (pyruvate dehydrogenase) autoantibodies in autoimmune rheumatic diseases. J Clin Immunol 12(3):201-209

29. Mouritsen S, Demant E, Permin H, Wiik A (1986) High prevalence of anti-mitochondrial antibodies among patients with some well-defined connective tissue diseases. Clin Exp Immunol 66(1):68-76

30. Koga T, Migita K, Miyashita T et al (2008) Determination of anticyclic citrullinated peptide antibodies in the sera of patients with liver diseases. Clin Exp Rheumatol 26(1):121-124 
31. Santiago M, Baron M, Miyachi K et al (2008) A comparison of the frequency of antibodies to cyclic citrullinated peptides using a third generation anti-CCP assay (CCP3) in systemic sclerosis, primary biliary cirrhosis and rheumatoid arthritis. Clin Rheumatol 27(1):77-83

32. Walker EJ, Hirschfield GM, Xu C et al (2009) CTLA4/ICOS gene variants and haplotypes are associated with rheumatoid arthritis and primary biliary cirrhosis in the Canadian population. Arthritis Rheum 60(4):931-937
33. Meyers RP, Shaheen AA, Swain MG et al (2007) Rituximab for primary biliary cirrhosis (PBC) refractory to ursodeoxycholic acid (UDCA). Hepatology 46(Suppl 1):550A

34. Kawano A, Shimoda S, Kamihira T, Ishikawa F, Niiro H, Soejima Y, Taketomi A, Maehara Y, Nakamura M, Komori A, Migita K, Ishibashi H, Azuma M, Gershwin ME, Harada M (2007) Peripheral tolerance and the qualitative characteristics of autoreactive T cell clones in primary biliary cirrhosis. J Immunol 179 (5):3315-3324 